\title{
Activity-based Protein Profiling Approaches for Transplantation
}

\author{
Mario Navarrete, PhD, ${ }^{1,2}$ John A. Wilkins, PhD,,$^{1,2}$ Ying Lao, MSc, ${ }^{1,2}$ David N. Rush, MD, ${ }^{3}$ \\ Peter W. Nickerson, MD, ${ }^{1,3}$ and Julie Ho, MD ${ }^{1,2,3}$
}

\begin{abstract}
Enzyme activity may be more pathophysiologically relevant than enzyme quantity and is regulated by changes in conformational status that are undetectable by traditional proteomic approaches. Further, enzyme activity may provide insights into rapid physiological responses to inflammation/injury that are not dependent on de novo protein transcription. Activity-based protein profiling (ABPP) is a chemical proteomic approach designed to characterize and identify active enzymes within complex biological samples. Activity probes have been developed to interrogate multiple enzyme families with broad applicability, including but not limited to serine hydrolases, cysteine proteases, matrix metalloproteases, nitrilases, caspases, and histone deacetylases. The goal of this overview is to describe the overall rationale, approach, methods, challenges, and potential applications of ABPP to transplantation research. To do so, we present a case example of urine serine hydrolase ABPP in kidney transplant rejection to illustrate the utility and workflow of this analytical approach. Ultimately, developing novel transplant therapeutics is critically dependent on understanding the pathophysiological processes that result in loss of transplant function. ABPP offers a new dimension for characterizing dynamic changes in clinical samples. The capacity to identify and measure relevant enzyme activities provides fresh opportunities for understanding these processes and may help identify markers of disease activity for the development of novel diagnostics and real-time monitoring of patients. Finally, these insights into enzyme activity may also help to identify new transplant therapeutics, such as enzymespecific inhibitors.
\end{abstract}

(Transplantation 2019;103:1790-1798)

\section{TRANSPLANTATION AND HUMAN -OMICS}

The development of new therapeutics is a key unmet need in transplantation and critically dependent on improving our mechanistic understanding of the processes that lead

Received 25 January 2019. Revision received 19 March 2019

Accepted 28 March 2019.

${ }^{1}$ Manitoba Centre for Proteomics and Systems Biology, Winnipeg, MB, Canada.

${ }^{2}$ Department of Internal Medicine, Section Biomedical Proteomics, University of Manitoba, Winnipeg, MB, Canada.

${ }^{3}$ Department of Internal Medicine, Section Nephrology, University of Manitoba, Winnipeg, $M B$, Canada.

M.N., J.A.W., Y.L., D.N.R., P.W.N., and J.H. participated in the literature review, performing the research, and writing the article.

D.N.R is a consultant with Astellas Pharma, and P.W.N is a consultant with Astellas Pharma and Vitaeris Inc.

Serine hydrolase activity-based protein profiling research was supported by an operating grant from the Canadian Institutes of Health Research (\#287559). Cysteine protease activity-based protein profiling research was supported by the Canadian Donation and Transplantation Research Program (CDTRP). J.H. was supported by a Canadian Institutes of Health Research New Investigator Salary Award (\#340137). P.N. was supported by the Flynn Family Chair in Renal Transplantation.

Correspondence: Julie Ho, MD FRCPC, Departments of Internal Medicine and Immunology, Sections of Nephrology and Biomedical Proteomics, University of Manitoba, GE421C Health Sciences Centre, 820 Sherbrook St, Winnipeg, Manitoba R3A 1R9, Canada. (jho@hsc.mb.ca).

Copyright (C) 2019 Wolters Kluwer Health, Inc. All rights reserved.

ISSN: 0041-1337/19/10309-1790

DOI: $10.1097 /$ TP.0000000000002752 to graft loss. ${ }^{1-3}$ Such information could identify modifiable early events that cumulatively result in loss of graft function. Although animal models have been central to the identification of many fundamental biologically relevant processes, ${ }^{4}$ there is a clear and recognized need for more patient-derived data as the results of these model systems do not necessarily represent events most relevant to humans. Fitzgerald et $\mathrm{al}^{4}$ cogently argued that a human phenomic science approach can accelerate personalized medicine and improve clinical trial design through in-depth human phenotyping to identify mechanisms of action, toxicities, and responses to relevant clinical outcomes. However, delineating the underlying functional relevance of -omic data is a key to its interpretation and foundational to the accurate phenotyping of humans in health and disease.

There are numerous examples in which understanding the spectrum from genes to downstream effector molecules has led to key clinical innovations in transplantation. High-resolution typing has significantly improved our understanding of allorecognition, and HLA class II molecular mismatch assessment can be used to inform personalized risk for the development of de novo donorspecific antibody, T-cell-mediated rejection (TCMR), antibody-mediated rejection (AMR), and graft loss..$^{5-7}$ Transplant biopsy transcriptomics improves the diagnostic precision for AMR and can identify patients at high risk of graft loss. ${ }^{8-10}$ Peripheral blood and urine transcriptomics and urine metabolomics have each yielded potential 


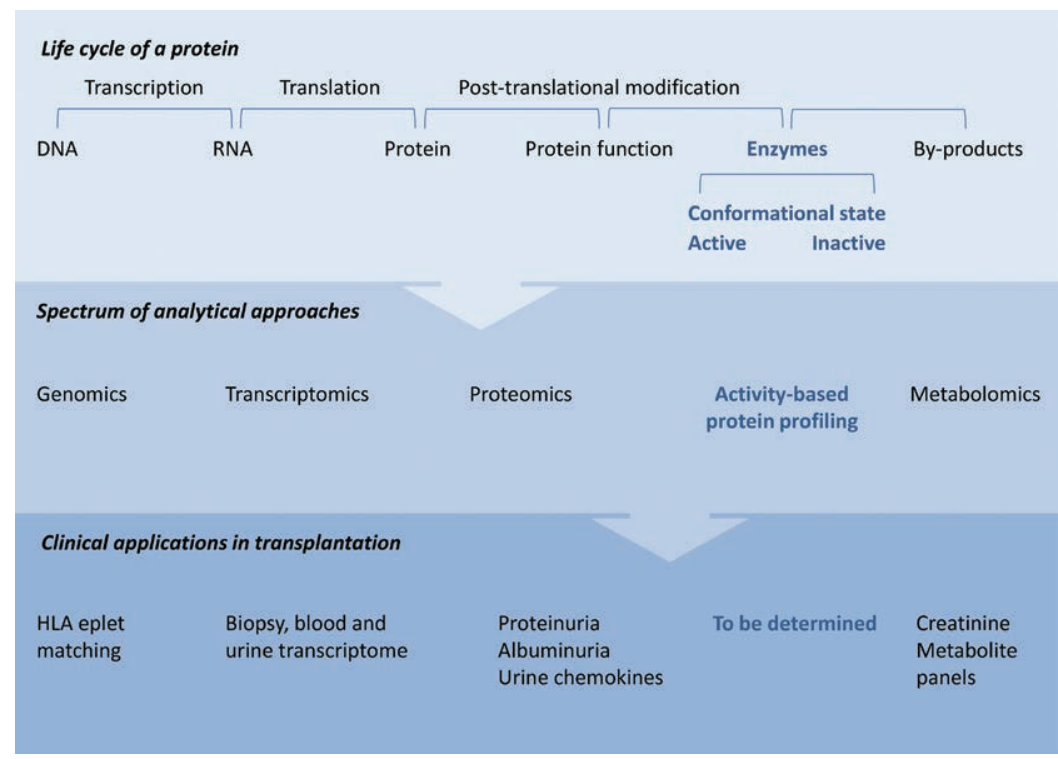

FIGURE 1. From genes to downstream effector molecules in transplantation. The spectrum of unbiased -omic approaches can be used to characterize different stages of a protein. Activity-based protein profiling is a proteomic technique to identify enzymes in an active conformational state and may provide important new insights into transplantation.

noninvasive biomarker panels for subclinical and clinical rejection. ${ }^{11-15}$ Within the genes to downstream effector molecules paradigm, proteomics falls between transcriptomics and metabolomics and offers complementary analytical approaches (Figure 1).

Comparative analysis and integration of data from such complementary approaches provide an additional level of confidence in candidate pathways, such as when transcript and protein changes fully align. For example, mRNA levels of granzyme B and its associated inhibitor serine protease inhibitor-9 was found to be associated with clinical biopsy-proven rejection urine samples. ${ }^{16}$ Serine protease inhibitor-9 protein expression was subsequently shown to be increased in subclinical versus clinical rejection biopsies and correlated with granzyme B-positive T lymphocytes. ${ }^{17}$ Finally, the murine homolog of serine protease inhibitor- 9 (serine protease inhibitor-6) was demonstrated to be renoprotective during allograft inflammation by inhibiting the activity of granzyme B. ${ }^{18}$ Taken together, these data show excellent concordance between mRNA, protein expression, and activity data, and concordance between human and animal findings.

However, 1 of the key limitations to interpreting human genomic and transcriptomic data is the often unclear relationship between these upstream analytical approaches and their potential downstream relevance. Nucleic acidbased reporters do not necessarily correlate with protein expression levels, as multiple downstream mechanisms exist to control expression levels and functionality. Ultimately, it is proteins/metabolites that mediate biological processes, and their functional status reflects the direct manifestations and activity of genomic expression in real time. For example, in an adult cardiac surgery model of renal ischemia-reperfusion injury, we demonstrated that there are increased intraoperative urine serine hydrolase enzyme activities that preceded changes in protein quantity in patients who subsequently developed postoperative acute kidney injury. These observations were consistent with early intraoperative enzyme activity changes that preceded protein transcription/translation. ${ }^{19}$

Enzymes are proteins that catalyze the chemical reactions required for physiological function. A review of the Uniprot protein database indicates that $>3400$ entries are associated with enzymatic activity, highlighting the importance of this category of proteins. Many enzymes are maintained in a latent state until their functional activity is required (eg, complement activation, coagulation system). This is done to minimize possible undesired deleterious effects of unregulated activity. The transition to an active state may be a consequence of changes in conformation induced by posttranslational modifications, acquisition of cofactors, or proteolytic cleavage. Such activity changes are independent of changes in the absolute quantity of a given enzyme and provide the ability for systems to rapidly respond to changes in their environment. Thus, the presence of an enzyme does not necessarily indicate that it is in an active conformational state. Therefore, measurement of enzyme activity is a critical component in understanding dynamic clinical conditions, and this may be more pathophysiologically relevant than absolute quantification of enzyme amounts. Unfortunately, most antibody and mass spectrometry-based analytical approaches used in proteomics do not provide information on its activity or functional status, which stresses the need for complementary approaches. Activity-based protein profiling (ABPP) was developed as an approach to address this need.

\section{ACTIVITY-BASED PROTEIN PROFILING}

ABPP is designed to specifically detect the active forms of an enzyme. The approach is based on the premise that only active enzymes are capable of interacting with their substrates. ${ }^{20}$ Thus the selective reaction of a sample with an activity probe displaying chemical properties similar to their cognate substrate offers a means of specifically binding the active species of enzyme. As enzymes in a given family share a common mechanism of catalysis, it is possible 
to use a single probe to simultaneously identify multiple active types of enzymes within a single family. The basic design of an activity probe incorporates a reactive group which provides the specificity for a given set of enzyme targets, a spacer, and a tag which may directly function as a reporter (eg, fluorescence) or for affinity capture (eg, biotin). The reactive groups are often specific irreversible inhibitors that covalently bind to the catalytic site of active enzymes, providing a stable association between the probe and enzymes. The tags allow for the direct or indirect visualization of the labeled enzymes in situ or gel-separated proteins. The tags also offer a basis for the affinity purification of labeled enzymes, which in conjunction with liquid chromatography-tandem mass spectrometry can be used for identifying the labeled enzymes. ${ }^{21}$ The tags can be directly incorporated into the probe or subsequently coupled to the reacted probe using click chemistry. ${ }^{22}$ The click chemistry approach offers the potential of functionalizing aliquots of a single probe-labeled sample for reaction with different reporters for comparative proteomics. ABPP complements quantitative proteomics by reporting on enzyme functional status. ${ }^{20,21}$

The use of ABPP has rapidly expanded in the last 10-15 years with the generation of an extensive and growing set of activity probes for interrogating different enzymes or enzyme families ${ }^{23-32}$ (Table 1). ABPP has led to the identification of novel therapeutic targets in diverse disease systems ranging from cancer biology to microbiology. ${ }^{33-37}$ Using serine hydrolase ABPP, the Cravatt group demonstrated increased KIAA1363 and monoacylglycerol lipase activity associated with increased aggressiveness of cancer cell lines. The inhibition of these enzymes resulted in decreased tumor growth in mouse models. ${ }^{20,33,34}$ Using a combination of serine hydrolase and cysteine protease ABPP, the Bogyo group found increased falcipain 1 activity during the invasive merozoite stage of malaria and characterized regulators of erythrocyte rupture to identify new antimalarial drug targets. ${ }^{35,36,38}$

ABPP can also be used to identify novel enzyme biomarkers of disease activity, which can then be tailored to enzymespecific activity assays. ${ }^{39}$ One of the major challenges in proteomics has been characterizing low-abundance proteins, which can be masked by high-abundance proteins in mass spectrometry-based analyses. However, enzymespecific substrates can be used to amplify the signals from active enzymes irrespective of their quantity within a complex biological sample. This signal amplification offers the potential to develop highly sensitive enzyme activity assays for noninvasive biomarker assays. Additionally, activity-based probes have been developed for in vivo imaging approaches of disease activity. For example, the Bogyo group developed a caspase activity probe for in vivo imaging in a mouse model of colorectal cancer to identify early disease activity and monitor response to therapy. ${ }^{37}$ Taken together, these chemical proteomic approaches offer a diverse range of effective tools that can be used to interrogate systems relevant to transplantation.

\section{APPLICATION OF ABPP IN TRANSPLANTATION}

To illustrate the approach, interpretation, and potential challenges in the application of ABPP, we present a selected case example of serine hydrolase ABPP analysis in kidney transplant subclinical and clinical TCMR. Overall, the ABPP approach involves labeling of samples with an activity-based probe specific to the enzyme family of interest using reporter tags to obtain different readouts. An example of the experimental workflow is depicted in Figure 2.

\section{Serine Hydrolase Enzyme Family}

The serine hydrolases share a serine centric charge relay system in their catalytic site. ${ }^{40}$ This property permits the targeting of many serine hydrolases with a single activity probe to profile a broad range of activities. The serine hydrolase family constitutes $\sim 1 \%$ of predicted protein products of the eukaryotic genome and includes $>100$ serine proteases and $\sim 10$ peptidases, amidases, esterases, and lipases. ${ }^{41}$ Active urinary serine hydrolases, such as tissue kallikrein, urokinase-type plasminogen activator and plasmin have been identified in healthy individuals and may be involved with normal electrolyte homeostasis. ${ }^{42}$ Although many members are well characterized (eg, trypsin, elastase,

\section{TABLE 1.}

Activity-based probe examples: enzyme families

\begin{tabular}{|c|c|c|}
\hline Enzyme family & Application & References \\
\hline Serine hydrolases & Profiling of tissue activity & Liu et $a^{23}$ \\
\hline Thiolase & Activity profiling and enzyme purification & Adam et $\mathrm{al}^{24}$ \\
\hline \multicolumn{3}{|l|}{ Aldehyde dehydrogenase } \\
\hline \multicolumn{3}{|l|}{ NAD/NADP-dependent oxidoreductase } \\
\hline \multicolumn{3}{|l|}{ Enoyl CoA hydratase } \\
\hline \multicolumn{3}{|l|}{ Epoxide hydrolase } \\
\hline \multicolumn{3}{|l|}{ Glutathione S-transferase } \\
\hline Histone deacetylases & HDAC complex profiling & Salisbury and Cravatt ${ }^{25}$ \\
\hline Cytochrome P450 & Analysis of hepatic responses & Wright and Cravatt, ${ }^{26}$ Wright et al ${ }^{28}$ \\
\hline Nitrilases & Active site mapping & Barglow et $\left.a\right|^{27}$ \\
\hline Matrix metalloproteinases & In vivo dynamics & Keow et $\mathrm{al}^{29}$ \\
\hline Cysteine cathepsin proteases & Inhibitor analysis & Falgueyret et al ${ }^{30}$ \\
\hline Cysteine proteases & Functional analysis of enzyme in families & Kato et $\mathrm{al}^{31}$ \\
\hline Nonribosomal peptide synthetases & Antibiotic development & |shikawa et al ${ }^{32}$ \\
\hline
\end{tabular}

CoA, coenzyme A; HDAC, histone deacetylase; NAD, nicotinamide adenine dinucleotide; NADP, nicotinamide adenine dinucleotide phosphate. 


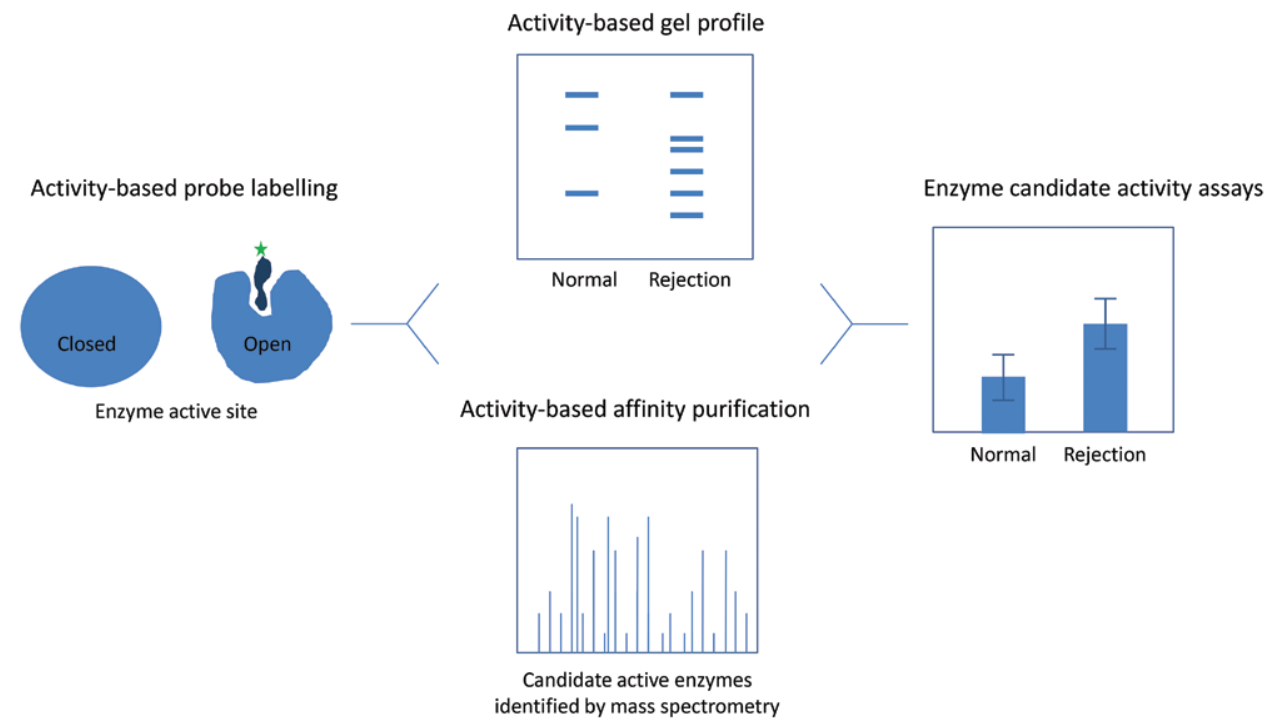

FIGURE 2. Outline of a typical activity-based protein profiling experiment. Samples are reacted with probes which covalently label active enzymes of the target family. The labeled proteins can be separated by sodium dodecyl sulfate-polyacrylamide gel electrophoresis and visualized or affinity purified and identified using mass spectrometry. The development of enzyme-specific quantitative activity assays offers the potential for validation and high-throughput analysis of patient samples.

thrombin, acetylcholinesterase), the functions and physiological substrates of many have yet to be determined. Indeed, the distribution and roles of approximately $50 \%$ of the metabolic serine hydrolases remain unknown in humans. ${ }^{41}$

Several serine proteases and their cognate inhibitors have been implicated in subclinical and clinical rejection, such as granzymes $\mathrm{A} / \mathrm{B}$ and serine protease inhibitor- $9 .{ }^{16-18,43,44}$ More recently, it has been reported that granzyme $\mathrm{B}^{+}$regulatory $\mathrm{B}$ cells are increased in transplant patients, and it is suggested that these regulatory B cells may play a role in maintaining transplant tolerance and host defense. ${ }^{45}$ SERP-1 is a serine protease inhibitor that inhibits tissuetype and urokinase-type plasminogen activators, thrombin, factor Xa, and plasmin and has been shown to inhibit early injury and chronic allograft rejection in a rat renal transplant model. ${ }^{46}$ Finally, unbiased transcriptomic studies identified the serine hydrolase acyloxyacyl hydrolase as a candidate intragraft gene during TCMR, and it is one of numerous candidates currently under consideration by the Banff working group within a composite molecular endpoint. ${ }^{9}$ Taken together, these data formed a basis for evaluating serine hydrolase ABPP in subclinical and clinical rejection urines from kidney transplant patients.

\section{In-gel ABPP Analysis}

The purpose of the in-gel ABPP approach is to simultaneously profile the cumulative activities of the serine hydrolase enzyme family ( $>200$ enzymes). Serine hydrolase ABPP was undertaken in kidney transplant patients with normal histology and graft function, subclinical TCMR $\geq 1 \mathrm{~A}$ rejection, and clinical TCMR $\geq 1 \mathrm{~A}$ in a previously described discovery proteomics cohort. ${ }^{47}$ Urines were reacted with a fluorescent-tagged serine hydrolase activity probe (fluorophosphonate-TAMRA, FP-TAMRA), and probe-labeled samples were separated by SDS-PAGE using previously described methods. ${ }^{19,42}$ An illustrative example of the serine hydrolase activities in normal transplant, subclinical, and clinical rejection urines is shown
(Figure 3A). This example demonstrates differences in the activity bands between the 3 patient groups in complexity and intensity and the presence of interindividual variability within each group. The changes in activity bands were most notable in the subclinical rejection patients, whereas the clinical rejection patients showed the greatest reduction in overall serine hydrolase activity (Figure 3A). The normal transplant patients had additional urine serine hydrolase activity bands that were not previously observed in healthy individuals. ${ }^{42}$

The activity-based gel profile enables rapid visual comparison between disease states and offers the basis for identifying shared features within a group by providing information on the patterns, apparent molecular weights, and relative activities of the serine hydrolase enzyme family. Alterations in the patterns of labeling may reflect changes in the overall urinary enzyme composition or differential activation of enzymes already present. The intensity of the fluorescence staining can provide an indirect measure of relative activities in a given gel region if the probe is not limiting and signals are not saturating. However, it is important to consider that a single gel band may not necessarily represent a single enzyme, as there may be many different enzymes within the family with similar molecular weights. Thus, changes in band intensity can arise from the increased activity of a single enzyme or from the combined activities of multiple enzymes with similar molecular weights, and it is therefore not possible to extrapolate the activities of individual enzymes in the absence of enzymespecific activity assays. Despite these potential caveats, the in-gel analysis offers a rapid and convenient approach for comparing enzyme activity profiles independent of the observed intra and interindividual variability.

The activity-based gels can also be stained for total protein content to compare relative protein quantities versus enzyme activities in different molecular weight regions (eg, Coomasie blue, Sypro Ruby). Such information may provide indirect evidence for assessing the contributions of quantitative changes in enzyme levels to activity 

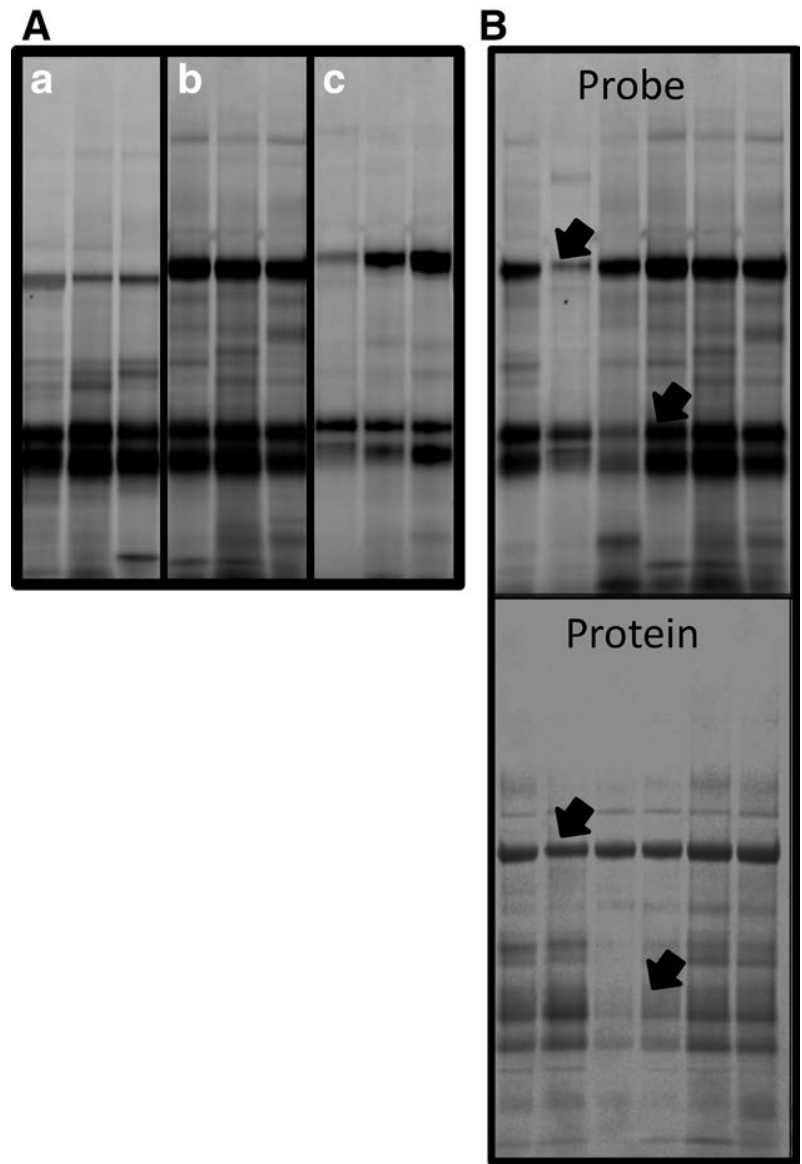

FIGURE 3. In-gel comparative activity-based protein profiling analysis. Urines from the indicated patient groups ( $n=3$ per group) were labeled with FP-TAMRA, separated by sodium dodecyl sulfatepolyacrylamide gel electrophoresis, and the labeled proteins visualized by fluorescence. A, (a) normal transplant, (b) subclinical TCMR $\geq$ Banff $1 \mathrm{~A} / \mathrm{B}$, and (c) clinical TCMR $\geq$ Banff $1 \mathrm{~A} / \mathrm{B}$. The individual bands indicate the active labeled serine hydrolase species in each urine. $B$, Comparison of the probe labeling (upper panel) and protein (lower panel) patterns in of the same urine sample. Note the lack of correlation between protein and probe levels in corresponding regions of the gel. Some examples of differences are indicated by arrows. FP-TAMRA, fluorophosphonate-TAMRA; TCMR, T-cell-mediated rejection.

differences. Indeed, enzyme activities may not directly correlate with enzyme quantity, emphasizing the critical importance of characterizing protein function. ${ }^{19,42}$ The illustrative example highlights 2 areas of activity that do not correlate with total protein levels in the corresponding regions of the gel (Figure 3B). This suggests that serine hydrolase enzymes detected by the activity probe may be low-abundance proteins and not necessarily the major proteins in the corresponding gel region.

Overall, this example highlights the utility of comparative in-gel ABPP for the analysis of urine samples. It is rapid and relatively simple and provides a basis for identifying differences between groups for an entire enzyme family. The method is readily adaptable for the analysis of clinical samples, as it requires $<100 \mu \mathrm{L}$ of urine.

\section{Identification of Active Enzymes}

The purpose of ABPP affinity purification is to identify the specific serine hydrolases present in an active form from all the potential candidates in the enzyme family (eg, serine hydrolases, $>200$ potential enzymes). Indeed, a limitation of the in-gel analysis is that it does not offer specific information regarding the identities of the probe-labeled proteins. However, the reporter tag in the activity probe can be used for affinity purification of active enzymes from a complex biological sample. The progression and effectiveness of this isolation can be monitored with in-gel analysis. Ultimately, the affinity-purified materials can be identified using mass spectrometry. ${ }^{19,42}$ As the patterns of probe reactivity may vary depending on the probe structure, it is beneficial to use probes with overlapping target enzyme repertoires to maximize coverage for in-depth enzyme identification. An example of the serine hydrolase activities that are common and unique to the FP-TAMRA and 6-N-biotinylaminohexyl isopropyl phosphorofluoridate (PF-biotin) activity probes in the same patient sample pool is illustrated in Figure 4A.

To illustrate the identification of specific active serine hydrolases using ABPP, urine pools from subclinical and clinical rejection patients were separately labeled with 2 activity probes with overlapping but nonidentical specificities (FP-TAMRA and PF-biotin). Pooled urine samples were used as the ABPP affinity purification/mass spectrometry experiments are a high-content, low-throughput analytical approach to identify active enzyme candidates from the total averaged population. The labeled proteins were affinity purified as previously described. ${ }^{19,42}$ Following affinity purification, there was an increase in the number and intensity of the labeled proteins with a marked reduction in the total protein content in the affinity-purified samples (Figure 4B). The in-gel patterns of the probe-labeled proteins in the starting materials were similar for subclinical and clinical rejection urines, with increased activities in the purified materials, and this type of result provides evidence for enrichment of the activity probe-labeled enzymes (Figure 4C). The increased "haze" in the $30-50 \mathrm{kDa}$ region may indicate a diverse range of low-abundance enzymes that have been enriched.

Tandem mass spectrometric analysis of the affinitypurified materials identified 33 serine hydrolases, the majority of which were detected in both subclinical and clinical rejection urines $(n=27)$. However, some enzymes were only detected in either subclinical or clinical rejection samples (Figure 4D). This type of analysis identifies specific active enzyme candidates. It is notable that the ABPP affinity purification may enable identification of low-abundance enzymes that might otherwise be undetectable using traditional proteomic approaches. Such approaches enable identification of physiologically relevant active enzymes irrespective of their quantitative abundance within complex biological samples. It is important to bear in mind that lack of activity probe labeling and mass spectrometry identification does not provide information regarding the presence or absence of the inactive enzyme, as this form will not react with the activity probe.

Disease states may arise from the emergence of novel enzyme activities, and ABPP can be integrated to provide downstream functional confirmation of differential transcriptomic or proteomic changes. However, it should be recognized that pathophysiological changes could occur via quantitative activity changes in the absence of compositional changes to those enzymes already present in normal samples. Such mechanisms enable rapid physiological responses to injury before the initiation of protein 
A Probe specificities

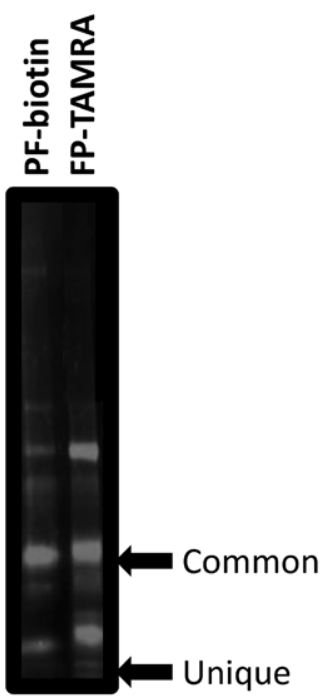

C PF-biotin
Clinical rejection

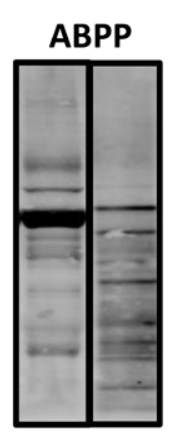

Start Purified
ABPP

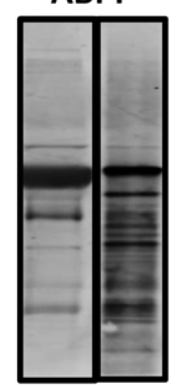

Start Purified
B FP-TAMRA

Subclinical

rejection

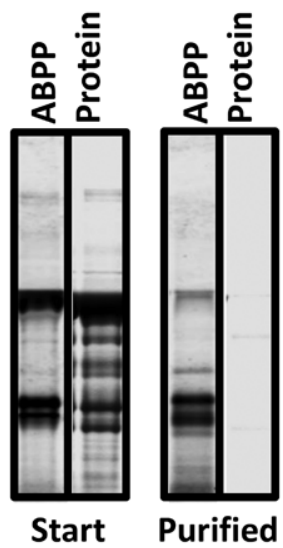

D Active enzymes identified

\section{Subclinical rejection}

FIGURE 4. Affinity purification and identification of active serine hydrolases. A, Probe specificities. A comparison of the FP-TAMRA and PF-biotin activity probe labeling of the same sample pool of patient urines. Overlapping areas of probe specificity and unique probe labeling are indicated by arrows. B, FP-TAMRA. A comparison of the FP-TAMRA activity probe labeling (ABPP) and protein (Protein) staining of starting material and purified material after affinity purification. Note the marked reduction in protein content of the purified material despite the clear capture of probe-labeled materials. C, PF-biotin. A comparison of the PF-biotin labeling of urine pools from patients undergoing subclinical and clinical rejection. Note the similarity in patterns between the 2 groups and the marked enrichment of labeled materials in the purified samples. D, Active enzymes identified. Venn diagram indicating the degree of overlap between the enzymes identified in the subclinical and clinical rejection urines. ABPP, activity-based protein profiling; FP-TAMRA, fluorophosphonateTAMRA; PF-biotin, 6-N-biotinylaminohexyl isopropyl phosphorofluoridate.

transcription and translation and further emphasize the necessity for functional protein assessment. ${ }^{19}$

\section{Validation Studies of Candidate Enzymes}

ABPP does not provide a direct measure of activity for individual enzymes, and therefore further studies are required to confirm ABPP findings. Validation approaches for ABPP candidates include evaluating enzyme-specific activity or using enzyme-specific inhibitors to knock down activity. Quantitative enzyme-specific activity assays can be used for high-throughput testing of individual candidates. These enzyme-specific activity assays are useful to explore the intra and interindividual heterogeneity and clinical confounders of enzyme activity in large clinical cohorts.

Thus, an essential step to transitioning to large-scale screening is the selection of enzyme candidates for the development of enzyme-specific activity assays. Selecting enzyme candidates for further testing may be guided by different considerations, but first it should be confidently identified by mass spectrometry from probe-labeled samples. Unique candidates may be useful to differentiate between disease groups, whereas candidates common to disease groups may provide insight into overlapping conditions along the spectrum of pathophysiology. While the literature may guide enzyme candidate selection based on their physiological relevance, this approach can be limited by the lack of knowledge regarding the sources and roles of many human enzymes. Finally, supporting experiments that demonstrate parallel transcript or protein changes of the candidate may provide some guidance; however, this approach is limited by the potential for enzyme activity changes that are independent of changes in enzyme quantity. Understanding these potential caveats in candidate 

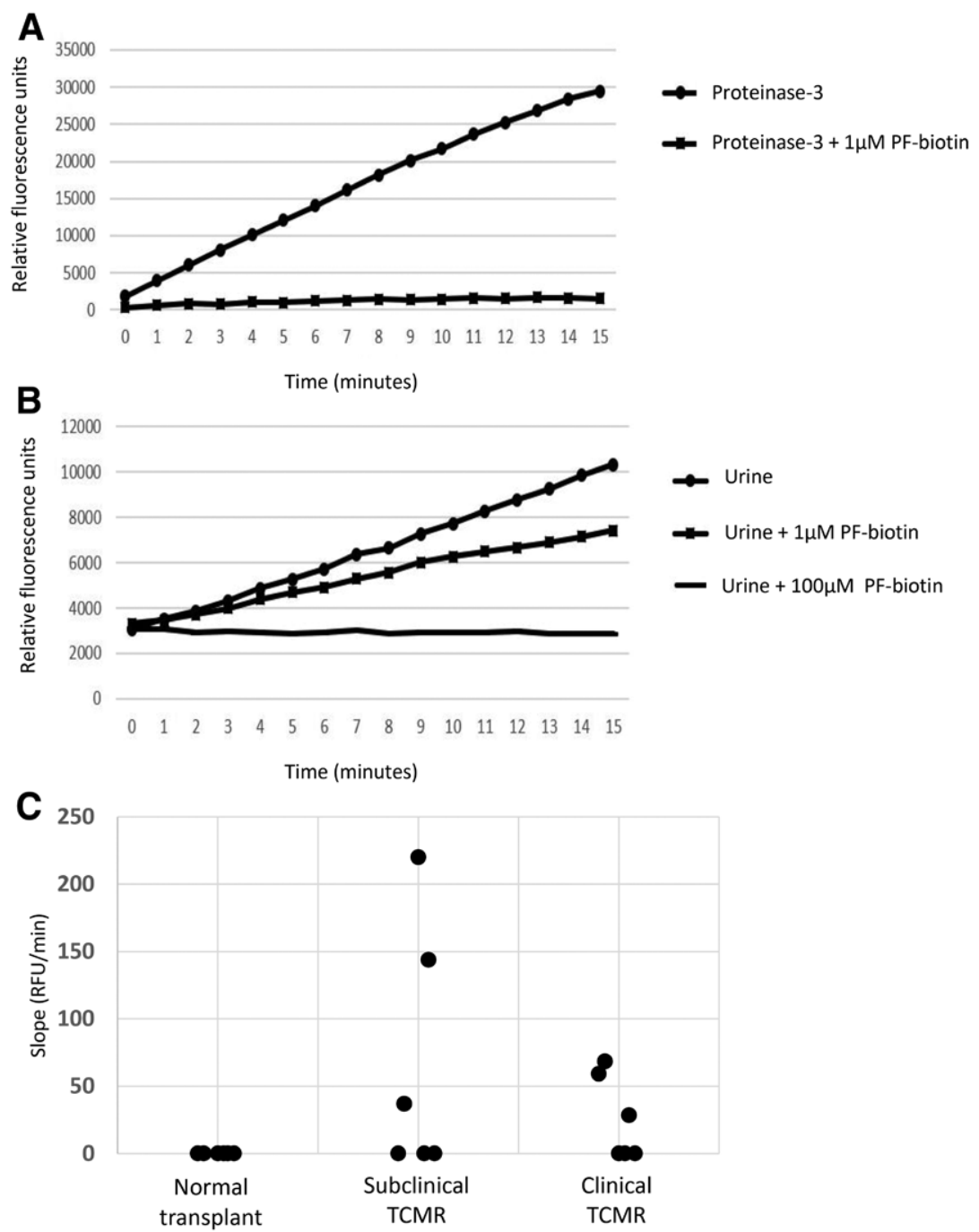

FIGURE 5. Development of an assay for proteinase-3 in urine. A, Demonstration of the kinetics of the response of recombinant proteinase-3 and inhibition of enzyme activity by PF-biotin probe. B, Detection of proteinase-3 activity in a rejection urine and dosedependent inhibition of the activity by PF-biotin probe. C, A comparison of proteinase-3 activity in urines from the indicated transplant patient groups ( $n=6$ per group). PF-biotin, $6-N$-biotinylaminohexyl isopropyl phosphorofluoridate; TCMR, T-cell-mediated rejection.

selection may also help in interpretation of the enzymespecific activity data.

In our illustrative example, the mass spectrometry results suggested that proteinase-3 (PRTN3, myeloblastin) activity was uniquely present in the subclinical rejection group compared with the clinical rejection group and healthy individuals and acute kidney injury patients. . $^{19,42}$ Therefore, a urine PRTN3 activity assay was adapted using a highly specific substrate ${ }^{48}$ and recombinant PRTN3 as a positive control. After optimization, the urine PRTN3 activity assay displayed good linearity and high sensitivity (Figure 5A). PRTN3 activity was fully inhibited by the PF-biotin probe, indicating that the enzyme(s) responsible for this reaction was a serine hydrolase (Figure 5B). Urine PRTN3 activity was then evaluated in a small, previously described discovery proteomics case-control cohort. ${ }^{47}$ Subclinical and clinical rejection patients demonstrated a trend to increased urine PRTN3 activity but not in patients with normal histology and graft function (Figure 5C). Urine PRTN3 activity was higher in 2 subclinical patients compared with clinical rejection patients, suggesting some overlap in the subclinical and clinical rejection phenotypes.
PRTN3 is a protease that is a highly expressed component in azurophilic granules of neutrophils; however, monocytes also normally express this enzyme in their lysosomes but at significantly lower levels. ${ }^{49}$ Interestingly, PRTN3 transcription may be regulated in part by steroidresponsive elements as it becomes highly expressed in CD4 $\mathrm{T}$ cells following treatment with methylprednisolone. ${ }^{50}$ These data raise the possibility of neutrophil, monocyte, CD4 $\mathrm{T}$ cell involvement, or an alternate cellular source of this enzyme during rejection. Clearly, this requires more comprehensive analysis to determine if there is an association between PRTN3 activity and graft status and to characterize the source and relevance of its activity.

This case example demonstrates the ABPP workflow from unbiased analysis to candidate validation including the development of enzyme-specific activity assays that enable screening of large clinical cohorts (Figure 2). These substrate-based assays can amplify activity signals from low-abundance enzymes and may be translatable into clinical tests. The challenges of developing and optimizing enzyme-specific activity assays include the potential for cross-substrate specificity, because enzymes within the same 
family share a similar catalytic site. This is the inverse of the strength of the ABPP approach, which leverages this similar mechanism of catalysis within an enzyme family. The availability of novel substrate screening libraries may be useful in addressing this issue for identification of enzyme-specific substrates. ${ }^{51,52}$ Despite these very real challenges, it is clear that enzyme-specific assays can be developed and have become an integral part of clinical monitoring programs, such as the ADAMTS13 activity assay for evaluation of thrombotic thrombocytopenic purpura. ${ }^{53}$

\section{CONSIDERATIONS REGARDING THE APPLICATION OF ABPP TO URINE}

As ABPP detects enzyme activity, it is essential that urine samples are collected in a manner that retains enzyme functionality. In our experience, routine collection with urine samples kept at $4^{\circ} \mathrm{C}$ or on ice until processing to obtain urine supernatants and storage at $-80^{\circ} \mathrm{C}$ has proven to be effective. Under these conditions, activity appears to be quite stable, as active enzymes have been detected in samples stored for $>10$ years. Some enzymes such as PRTN3 retain full activity after 7 freeze-thaw cycles (unpublished data). However, different enzymes may display different stabilities, so urine samples are aliquoted and stored to minimize freeze-thaw cycles, and individual candidate activities need to be confirmed.

Urine may not be the optimal environment for the activity of a particular enzyme, as it may have originated from different cellular and tissue compartments with distinct $\mathrm{pH}$ and ionic conditions. However, it is important to recall that the reactivity of ABPP probes is dependent on accessibility to the active site of a functional enzyme. Thus, labeling of active enzymes can occur even under suboptimal enzyme reaction conditions. Because different probes have distinct $\mathrm{pH}$ optima, labeling conditions should be optimized for each probe and enzyme-specific assay. The levels of nonspecific labeling can be assessed by monitoring the residual fluorescence patterns of samples that have been denatured before reaction with the probes.

Proteinuria does not interfere with activity-probe labeling of enzymes, because nonactive proteins do not compete for the probe. However, samples with high protein levels may complicate in-gel analysis because of band distortion due to high-abundance proteins such as albumin. There may be conditions in which urine contains natural noncompetitive inhibitors. These could potentially interfere with ABPP, and it may be beneficial to remove lowmolecular weight species by dialysis or gel permeation before labeling. However, this has not been our general practice, as we seek to characterize which enzymes are active under physiological conditions in the presence of endogenous inhibitors. Finally, ABPP is broadly applicable to many different sample types, including blood, tissue, cell culture supernatants, and lysates; and the optimal sample handling and activity probe labeling conditions require optimization for different experimental systems.

\section{SUMMARY - IMPLICATIONS FOR TRANSPLANTATION RESEARCH}

Developing novel transplant therapeutics is critically dependent on understanding the key pathophysiological processes that result in loss of transplant function in humans. ABPP provides an unbiased simultaneous measure of the functional status of enzymes within an enzyme family. This offers a unique adjunct to other high-content analytics such as genomics, transcriptomics, proteomics, or metabolomics, with the benefit of providing real-time information. ABPP can facilitate sequential sample analysis for detailed temporal mapping of dynamic clinical changes.

Changes in enzyme activity may be associated with the development of rejection or compensatory mechanisms in response to injury. Clearly such information may help identify potential therapeutic targets for inhibition or enhancement. Some enzyme activities may ultimately serve as markers of disease activity, and the development of enzyme-specific activity assays makes this feasible. Rapid, noninvasive colorimetric urine test strips may enable routine screening or home-based testing for disease activity or ongoing inflammation/injury (eg, urinary leukocyte esterase test). ${ }^{54}$ Apart from patient convenience, this has important implications for populations who are distant to their transplant center. Finally, even if a given enzyme does not prove to be a viable biomarker, knowledge of the types and kinetics of enzyme changes in transplant patients may offer the potential to understand the biology of inductive events and a basis to more accurately subclassify or stage patients. In conclusion, by improving our understanding of the dynamic biochemical responses in renal transplant patients, ABPP may contribute to the development of novel diagnostics and therapeutics and help advance patient and graft outcomes.

\section{REFERENCES}

1. O'Connell PJ, Kuypers DR, Mannon RB, et al. Clinical trials for immunosuppression in transplantation: the case for reform and change in direction. Transplantation. 2017;101:1527-1534.

2. Stegall MD, Morris RE, Alloway RR, et al. Developing new immunosuppression for the next generation of transplant recipients: the path forward. Am J Transplant. 2016;16:1094-1101.

3. Neuberger JM, Bechstein WO, Kuypers DR, et al. Practical recommendations for long-term management of modifiable risks in kidney and liver transplant recipients: a guidance report and clinical checklist by the consensus on managing modifiable risk in transplantation (COMMIT) group. Transplantation. 2017;101(4S Suppl 2): S1-S56.

4. FitzGerald G, Botstein D, Califf R, et al. The future of humans as model organisms. Science. 2018;361:552-553.

5. Wiebe C, Pochinco D, Blydt-Hansen TD, et al. Class ॥ HLA epitope matching-A strategy to minimize de novo donor-specific antibody development and improve outcomes. Am J Transplant. 2013;13:3114-3122

6. Wiebe C, Rush DN, Nevins TE, et al. Class II eplet mismatch modulates tacrolimus trough levels required to prevent donor-specific antibody development. J Am Soc Nephrol. 2017;28:3353-3362.

7. Wiebe C, Ho J, Gibson IW, et al. Carpe diem-time to transition from empiric to precision medicine in kidney transplantation. Am J Transplant. 2018;18:1615-1625.

8. O'Connell PJ, Zhang W, Menon MC, et al. Biopsy transcriptome expression profiling to identify kidney transplants at risk of chronic injury: a multicentre, prospective study. Lancet. 2016;388:983-993.

9. Haas M, Loupy A, Lefaucheur C, et al. The Banff 2017 kidney meeting report: revised diagnostic criteria for chronic active $T$ cell-mediated rejection, antibody-mediated rejection, and prospects for integrative endpoints for next-generation clinical trials. Am J Transplant. 2018;18:293-307.

10. Sellarés J, Reeve J, Loupy A, et al. Molecular diagnosis of antibodymediated rejection in human kidney transplants. Am J Transplant. 2013;13:971-983. 
11. Roedder S, Sigdel T, Salomonis N, et al. The KSORT assay to detect renal transplant patients at high risk for acute rejection: results of the multicenter AART study. PLoS Med. 2014;11:e1001759.

12. Friedewald JJ, Kurian SM, Heilman RL, et al; Clinical Trials in Organ Transplantation 08 (CTOT-08). Development and clinical validity of a novel blood-based molecular biomarker for subclinical acute rejection following kidney transplant. Am J Transplant. 2019;19:98-109.

13. Suthanthiran M, Schwartz JE, Ding R, et al; Clinical Trials in Organ Transplantation 04 (CTOT-04) Study Investigators. Urinary-cell mRNA profile and acute cellular rejection in kidney allografts. N Engl J Med. 2013:369:20-31.

14. Suhre K, Schwartz JE, Sharma VK, et al. Urine metabolite profiles predictive of human kidney allograft status. J Am Soc Nephrol. 2016;27:626-636.

15. Ho J, Sharma A, Mandal R, et al. Detecting renal allograft inflammation using quantitative urine metabolomics and CXCL10. Transplant Direct. 2016;2:e78.

16. Muthukumar T, Ding R, Dadhania D, et al. Serine proteinase inhibitor-9, an endogenous blocker of granzyme B/perforin Iytic pathway, is hyperexpressed during acute rejection of renal allografts. Transplantation. 2003;75:1565-1570.

17. Rowshani AT, Florquin S, Bemelman F, et al. Hyperexpression of the granzyme B inhibitor PI-9 in human renal allografts: a potential mechanism for stable renal function in patients with subclinical rejection. Kidney Int. 2004;66:1417-1422.

18. Lau A, Khan K, Pavlosky A, et al. Serine protease inhibitor-6 inhibits granzyme B-mediated injury of renal tubular cells and promotes renal allograft survival. Transplantation. 2014;98:402-410.

19. Navarrete M, Ho J, Dwivedi RC, et al. Activity-based protein profiling of intraoperative serine hydrolase activities during cardiac surgery. $J$ Proteome Res. 2018;17:3547-3556.

20. Barglow KT, Cravatt BF. Activity-based protein profiling for the functional annotation of enzymes. Nat Methods. 2007;4:822-827.

21. Cravatt BF, Wright AT, Kozarich JW. Activity-based protein profiling: from enzyme chemistry to proteomic chemistry. Annu Rev Biochem. 2008;77:383-414.

22. Speers AE, Adam GC, Cravatt BF. Activity-based protein profiling in vivo using a copper(l)-catalyzed azide-alkyne [3+2] cycloaddition. $J$ Am Chem Soc. 2003;125:4686-4687.

23. Liu Y, Patricelli MP, Cravatt BF. Activity-based protein profiling: the serine hydrolases. Proc Natl Acad Sci U S A. 1999;96:14694-14699.

24. Adam GC, Sorensen EJ, Cravatt BF. Proteomic profiling of mechanistically distinct enzyme classes using a common chemotype. Nat Biotechnol. 2002;20:805-809.

25. Salisbury CM, Cravatt BF. Activity-based probes for proteomic profiling of histone deacetylase complexes. Proc Natl Acad Sci U S A. 2007;104:1171-1176.

26. Wright AT, Cravatt BF. Chemical proteomic probes for profiling cytochrome p450 activities and drug interactions in vivo. Chem Biol. 2007; 14:1043-1051

27. Barglow KT, Saikatendu KS, Bracey MH, et al. Functional proteomic and structural insights into molecular recognition in the nitrilase family enzymes. Biochemistry. 2008;47:13514-13523.

28. Wright AT, Song JD, Cravatt BF. A suite of activity-based probes for human cytochrome P450 enzymes. J Am Chem Soc. 2009;131: 10692-10700.

29. Keow JY, Pond ED, Cisar JS, et al. Activity-based labeling of matrix metalloproteinases in living vertebrate embryos. PLOS ONE. 2012;7:e43434.

30. Falgueyret JP, Black WC, Cromlish W, et al. An activity-based probe for the determination of cysteine cathepsin protease activities in whole cells. Anal Biochem. 2004;335:218-227.

31. Kato $D$, Boatright KM, Berger AB, et al. Activity-based probes that target diverse cysteine protease families. Nat Chem Biol. 2005;1: 33-38.
32. Ishikawa F, Tanabe G, Kakeya H. Activity-based protein profiling of non-ribosomal peptide synthetases. Curr Top Microbiol Immunol. 2019;420:321-349

33. Jessani N, Liu Y, Humphrey M, et al. Enzyme activity profiles of the secreted and membrane proteome that depict cancer cell invasiveness. Proc Natl Acad Sci U S A. 2002;99:10335-10340.

34. Nomura DK, Long JZ, Niessen S, et al. Monoacylglycerol lipase regulates a fatty acid network that promotes cancer pathogenesis. Cell. 2010;140:49-61.

35. Greenbaum DC, Baruch A, Grainger M, et al. A role for the protease falcipain 1 in host cell invasion by the human malaria parasite. Science. 2002;298:2002-2006.

36. Arastu-Kapur S, Ponder EL, Fonović UP, et al. Identification of proteases that regulate erythrocyte rupture by the malaria parasite plasmodium falciparum. Nat Chem Biol. 2008:4:203-213.

37. Edgington LE, Berger AB, Blum G, et al. Noninvasive optical imaging of apoptosis by caspase-targeted activity-based probes. Nat Med. 2009;15:967-973.

38. Winzeler EA. Malaria research in the post-genomic era. Nature. 2008 455:751-756

39. Vizovišek M, Vidmar R, Drag M, et al. Protease specificity: towards in vivo imaging applications and biomarker discovery. Trends Biochem Sci. 2018;43:829-844.

40. Blow DM, Birktoft JJ, Hartley BS. Role of a buried acid group in the mechanism of action of chymotrypsin. Nature. 1969;221:337-340.

41. Simon GM, Cravatt BF. Activity-based proteomics of enzyme superfamilies: serine hydrolases as a case study. J Biol Chem. 2010;285: 11051-11055.

42. Navarrete $\mathrm{M}, \mathrm{Ho} \mathrm{J}$, Krokhin $\mathrm{O}$, et al. Proteomic characterization of serine hydrolase activity and composition in normal urine. Clin Proteomics. 2013;10:17.

43. van Ham SM, Heutinck KM, Jorritsma T, et al. Urinary granzyme A mrna is a biomarker to diagnose subclinical and acute cellular rejection in kidney transplant recipients. Kidney Int. 2010;78:1033-1040.

44. Li B, Hartono C, Ding R, et al. Noninvasive diagnosis of renal-allograft rejection by measurement of messenger RNA for perforin and granzyme B in urine. N Engl J Med. 2001;344:947-954.

45. Zhu J, Zeng Y, Dolff S, et al. Granzyme B producing B-cells in renal transplant patients. Clin Immunol. 2017;184:48-53.

46. Bédard EL, Jiang J, Arp J, et al. Prevention of chronic renal allograft rejection by SERP-1 protein. Transplantation. 2006;81:908-914.

47. Ho J, Rush DN, Krokhin O, et al. Elevated urinary matrix metalloproteinase-7 detects underlying renal allograft inflammation and injury. Transplantation. 2016;100:648-654.

48. Korkmaz B, Attucci S, Juliano MA, et al. Measuring elastase, proteinase 3 and cathepsin $G$ activities at the surface of human neutrophils with fluorescence resonance energy transfer substrates. Nat Protoc. 2008;3:991-1000

49. Brunini F, Page TH, Gallieni M, et al. The role of monocytes in ANCAassociated vasculitides. Autoimmun Rev. 2016;15:1046-1053.

50. De Andres C, García Ml, Goicoechea H, et al. Genes differentially expressed by methylprednisolone in vivo in CD4 T lymphocytes from multiple sclerosis patients: potential biomarkers. Pharmacogenomics J. 2018;18:98-105

51. Poreba M, Salvesen GS, Drag M. Synthesis of a HyCoSuL peptide substrate library to dissect protease substrate specificity. Nat Protoc. 2017; 12:2189-2214.

52. Kim M, Shin DS, Kim J, et al. Substrate screening of protein kinases: detection methods and combinatorial peptide libraries. Biopolymers. 2010;94:753-762.

53. Kokame K, Nobe Y, Kokubo Y, et al. FRETS-VWF73, a first fluorogenic substrate for ADAMTS13 assay. Br J Haematol. 2005;129:93-100.

54. Chernow B, Zaloga GP, Soldano S, et al. Measurement of urinary leukocyte esterase activity: a screening test for urinary tract infections. Ann Emerg Med. 1984;13:150-154. 